\title{
Transmission dynamics of a Huanglongbing model with cross protection
}

\author{
Lei Luo ${ }^{1}$, Shujing Gao ${ }^{1,2^{*}}$, Yangqiu $\mathrm{Ge}^{1}$ and Youquan Luo ${ }^{1}$
}

"Correspondence: gaosjmath@126.com

${ }^{1}$ Key Laboratory of Jiangxi Province for Numerical Simulation and Emulation Techniques, Gannan Normal University, Ganzhou, 341000, P.R. China

${ }^{2}$ National Research Center of Navel

Orange Engineering and

Technology, Gannan Normal University, Ganzhou, 341000, P.R. China

\section{Springer}

\begin{abstract}
Huanglongbing $(\mathrm{HLB})$ is one of the most common widespread vector-borne transmission diseases through psyllid, which is called a kind of cancer of plant disease. In recent years, biologists have focused on the role of cross protection strategy to control the spread of HLB. In this paper, according to transmission mechanism of HLB, a deterministic model with cross protection is formulated. A threshold value $R_{0}$ is established to measure whether or not the disease is uniformly persistent. The existence of a backward bifurcation presents a further sub-threshold condition below $R_{0}$ for the spread of the disease. We also discuss the effects of cross protection and removing infected trees in spreading the disease. Numerical simulations suggest that cross protection is a promotion control measure, and replanting trees is bad for HLB control.
\end{abstract}

Keywords: Huanglongbing model; cross protection; backward bifurcation; sub-threshold value

\section{Introduction}

Nowadays, Huanglongbing (HLB) is one of the most serious problems of citrus worldwide caused by the bacteria Candidatus Liberibacter spp., whose name in Chinese means "yellow dragon disease" [1]. The main symptoms on HLB include chlorosis of leaves, dieback and, in extreme cases, tree death. Additionally, infected trees develop fruit that is of poor quality and drops early, reducing yields of edible and marketable fruit from diseased trees [2]. The infected trees are usually destroyed or become unproductive in 5 to 8 years [1].

Most of the known plant viruses are transmitted by insect vectors. HLB, a destructive disease of citrus, can be transmitted by grafting from psyllid to citrus. The primary vector of the spread of the disease is the psyllid (Diaphorina Citri Kuwayama) [3].

In order to control HLB effectively, most of growers usually take the following measures: pesticides, tree removal, antibiotics [4], changes to tree spacing, natural enemies of psyllid. A few new intervention strategies are explored, including heat treatment [5], new tolerant or resistant tree stocks [6], nutrient additions [7], cross protection, intercropping [8]. Cross protection is one of biological methods. In recent years, cross protection is widely considered and applied in prevention and control of plant diseases.

Cross protection, first shown by McKinney [9] with tobacco mosaic virus (TMV), is a phenomenon whereby prior infection with one (protecting) plant virus will prevent or interfere with superinfection by another, usually related (challenging) virus [10]. In [11], the

(c) The Author(s) 2017. This article is distributed under the terms of the Creative Commons Attribution 4.0 International License (http://creativecommons.org/licenses/by/4.0/), which permits unrestricted use, distribution, and reproduction in any medium, provided you give appropriate credit to the original author(s) and the source, provide a link to the Creative Commons license, and indicate if changes were made. 
authors explored the cross protection between MAV (protecting) virus and PAV (challenging) virus in cereal, which belong to barley yellow dwarf viruses (BYDVs). By vaccinating M6 CTV strains (protecting) in citrus aurantif olia, Cui et al. [12] proved the obvious effect of cross protection on Bendizao mandarin. Van Vuuren et al. [13] studied the effect of cross protection on HLB of Africa by vaccinating multiple citrus recession viral strains. Hartung [14], who improved T36 CTV strains, described the resistance effect of cross protection on citrus HLB.

In recent years, some mathematical models on plant disease have been studied by many researchers (see [15-19]). Meng and Li [15] discussed the effect of cultural control on the healthy growth of the host plant. Local stability for the free periodic solution and persistence of the disease are key issues in the study of epidemic models. In fact, these issues are solved. In [16], Meng et al. illustrated that biological control may be a better way for pest management strategies by adopting a new mathematical model. Zhang et al. [17] proposed and compared two different control strategies in the model. In [18], Zhao et al. proposed a plant disease model with Markov conversion and impulsive toxicant input. Then thresholds of extinction and persistence in mean were obtained.

To the best of the authors' knowledge, there has been little work on plant disease models with cross protection (see [10, 20, 21]). Gao et al. [20] took seasonality into account and put forward a nonautonomous plant disease model with cross protection. The results showed that cross protection played an important role in controlling the spread of the challenging virus in plants. Zhang et al. [21] proposed a model to study cross protection between the viruses in 1999. Zhang and Holt [10] improved the model in [21], in which cross protection can occur both naturally and through artificial intervention. Our main purpose is to investigate the transmission of HLB between citrus tree and psyllid populations with cross protection and evaluate the effect of cross protection in controlling the spread of HLB.

To achieve the above goals, we formulate a HLB model with cross protection and analyze the dynamical behavior theoretically including a backward bifurcation. Recently, there have been a number of studies on a backward bifurcation in the epidemic literature, for example, see [22-26]. Garba et al. [22] considered a dengue model with standard incidence formulation undergoing the phenomenon of backward bifurcation. Ahmed et al. [23] modeled the spread and control of dengue with limited public health resources, which exhibited the phenomenon of backward bifurcation. Li et al. [24] constructed an SIR epidemic model with nonlinear incidence and treatment. The results show that a backward bifurcation occurs if the capacity is small, and there exist bistable endemic equilibria if the capacity is low [27].

The paper is organized as follows. We formulate our HLB model with cross protection in Section 2. In Section 3, we determine the existence and stability of equilibrium point of the model. Moreover, we prove the existence of a backward bifurcation around the disease-free equilibrium. In Section 4, we discuss the persistence of the disease. $\mathrm{Nu}$ merical simulation and discussion are given in Section 5.

\section{Model formulation}

We now study the impact of cross protection with vector as described above on the transmission dynamics and control strategies. We firstly formulate a deterministic model of HLB by considering citrus trees totalling $N_{h}$ in a grove, which we divide into four categories: healthy, infected with the protecting virus alone, infected with the HLB virus 


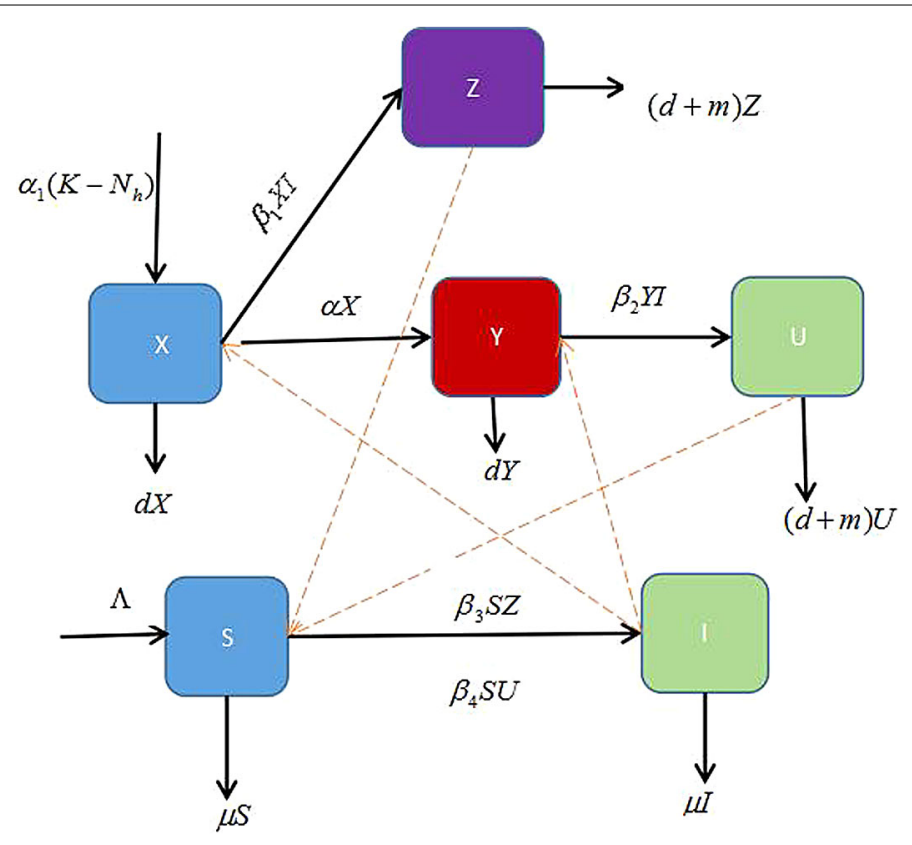

Figure 1 A schematic of the model system showing transitions to different categories for trees and psyllids. Black arrows show the transitions between compartments. Orange dashed arrows show the necessary interactions between trees and psyllids to obtain transmission.

(challenging) alone, and infected with both, designated as $X(t), Y(t), Z(t)$, and $U(t)$, respectively. Thus the total number of trees $N_{h}(t)=X(t)+Y(t)+Z(t)+U(t)$. We divide the vector population into a susceptible class $S(t)$ and an infected class $I(t)$. Let $N_{v}=S(t)+I(t)$ be the total number of psyllids. The model flow diagram is depicted in Figure 1. The model is a system of six ordinary differential equations:

$$
\left\{\begin{array}{l}
\frac{d X}{d t}=\alpha_{1}\left(K-N_{h}\right)-\alpha X-d X-\beta_{1} X I, \\
\frac{d Y}{d t}=\alpha X-d Y-\beta_{2} Y I, \\
\frac{d Z}{d t}=\beta_{1} X I-(d+m) Z, \\
\frac{d U}{d t}=\beta_{2} Y I-(d+m) U, \\
\frac{d S}{d t}=\Lambda-\beta_{3} S Z-\beta_{4} S U-\mu S, \\
\frac{d I}{d t}=\beta_{3} S Z+\beta_{4} S U-\mu I,
\end{array}\right.
$$

where $\alpha_{1}$ is the rate of replanting a citrus tree, $\alpha$ is the vaccination rate, $\beta_{1}$ is the probability that a susceptible tree becomes infected from contact with an infected psyllid with HLB virus, $\beta_{2}$ is the probability that a tree with protecting virus becomes infected from contact with an infected psyllid, $\beta_{3}$ is the probability that a susceptible psyllid becomes infected from contact with an infected tree, $\beta_{4}$ is the probability that a susceptible psyllid becomes infected from contact with an infected tree with two kinds of viruses, $\mu$ is the natural mortality of psyllid population, $m$ is the roguing rate of infected trees, $d$ is the natural death rate of a citrus tree, $\Lambda$ is the constant recruitment rate of a psyllid, $K$ is the maximum total number of citrus trees that can be planted in the grove. By the biological meaning, we assume that all parameters in model (2.1) are positive, and $\alpha \leq 1$ and $\alpha_{1} \leq 1$. 
The initial conditions for (2.1) are

$$
\begin{array}{rrr}
X(0) \geq 0, & Y(0) \geq 0, \quad Z(0) \geq 0, \\
U(0) \geq 0, \quad S(0) \geq 0, & I(0) \geq 0 .
\end{array}
$$

We will study the transmission dynamics of HLB disease in the rest of this paper. Before giving the main result, we present the following lemmas.

Lemma 2.1 Suppose $(X(t), Y(t), Z(t), U(t), S(t), I(t))$ is a solution of system (2.1) with initial conditions (2.2), then $(X(t), Y(t), Z(t), U(t), S(t), I(t)) \geq 0$ for all $t \geq 0$.

Lemma 2.2 Every solution $(X(t), Y(t), Z(t), U(t), S(t), I(t))$ of system (2.1) eventually enters

$$
\Omega=\left\{(X, Y, Z, U, S, I) \in R_{+}^{6}: 0 \leq X+Y+Z+U \leq \frac{\alpha_{1} K}{d+\alpha_{1}}, 0 \leq S+I \leq \frac{\Lambda}{\mu}\right\}
$$

and $\Omega$ is a positively invariant set for (2.1).

Proof From model (2.1), we get

$$
\frac{d N_{h}(t)}{d t}=\alpha_{1} K-\left(d+\alpha_{1}\right) N_{h}(t)-m(Z(t)+U(t))
$$

and

$$
\frac{d N_{v}(t)}{d t}=\Lambda-\mu N_{v}(t)
$$

Thus, $\frac{d N_{h}(t)}{d t} \leq 0$ if $N_{h}(t) \geq \frac{\alpha_{1} K}{d+\alpha_{1}}$, and $\frac{d N_{v}(t)}{d t} \leq 0$ if $N_{v}(t) \geq \frac{\Lambda}{\mu}$, which implies that $\Omega$ is positively invariant with respect to system (2.1). This completes the proof.

\section{Existence and stability of equilibrium points}

To better organize the analysis, in the following we denote $k_{1}=d+\alpha, k_{2}=d+\alpha_{1}, k_{3}=d+m$, $k_{4}=d+m+\alpha_{1}, k_{5}=d+\alpha+\alpha_{1}$, and $k_{6}=d+m+\mu$.

\subsection{Existence of equilibrium points}

In this subsection, we determine the existence of the equilibrium points of model (2.1). It is straightforward to establish that there is, for all parameter values, a disease-free equilibrium $E_{0}=\left(X_{0}, Y_{0}, Z_{0}, U_{0}, S_{0}, I_{0}\right)=\left(\frac{\alpha_{1} K d}{k_{1} k_{2}}, \frac{\alpha \alpha_{1} K}{k_{1} k_{2}}, 0,0, \frac{\Lambda}{\mu}, 0\right)$.

The stability of $E_{0}$ can be described by using the next generation operator method [28, 29]. According to the notation in [29], the Jacobian matrices $F$ (of new infection terms) and $V$ (of remaining transition terms) are given, respectively [30]. We have

$$
F=\left(\begin{array}{ccc}
0 & 0 & \beta_{1} X_{0} \\
0 & 0 & \beta_{2} Y_{0} \\
\frac{\beta_{3} \Lambda}{\mu} & \frac{\beta_{4} \Lambda}{\mu} & 0
\end{array}\right) \text { and } V=\left(\begin{array}{ccc}
k_{3} & 0 & 0 \\
0 & k_{3} & 0 \\
0 & 0 & \mu
\end{array}\right)
$$


Therefore, the basic reproductive number for system (2.1) is

$$
R_{0}=\rho\left(F V^{-1}\right)=\sqrt{\frac{\beta_{2} \beta_{4} \alpha \alpha_{1} K \Lambda+\beta_{1} \beta_{3} \alpha_{1} K \Lambda d}{\mu^{2} k_{1} k_{2} k_{3}}},
$$

here $\rho$ is the spectral radius of a matrix.

Now we turn to discussing a possible endemic equilibrium point $E^{*}\left(X^{*}, Y^{*}, Z^{*}, U^{*}, S^{*}, I^{*}\right)$ in the interior of the feasible region $\Omega$. Here $X^{*}, Y^{*}, Z^{*}, U^{*}, S^{*}, I^{*}>0$ satisfy the following equilibrium equations:

$$
\begin{aligned}
& \alpha_{1}\left(K-N_{h}^{*}\right)-\alpha X^{*}-d X^{*}-\beta_{1} X^{*} I^{*}=0, \\
& \alpha X^{*}-d Y^{*}-\beta_{2} Y^{*} I^{*}=0, \\
& \beta_{1} X^{*} I^{*}-(d+m) Z^{*}=0, \\
& \beta_{2} Y^{*} I^{*}-(d+m) U^{*}=0, \\
& \Lambda-\beta_{3} S^{*} Z^{*}-\beta_{4} S^{*} U^{*}-\mu S^{*}=0, \\
& \beta_{3} S^{*} Z^{*}+\beta_{4} S^{*} U^{*}-\mu I^{*}=0 .
\end{aligned}
$$

Adding (3.6) and (3.7), the coordinates of an endemic equilibrium point $\left(X^{*}, Y^{*}, Z^{*}, U^{*}\right.$, $\left.S^{*}, I^{*}\right)$ must satisfy

$$
S^{*}=\frac{\Lambda}{\mu}-I^{*}
$$

According to (3.3)-(3.7), we get

$$
Y^{*}=\frac{\alpha \mu^{2} k_{3}}{\beta_{1} \beta_{3}\left(\Lambda-\mu I^{*}\right)\left(d+\beta_{2} I^{*}\right)+\alpha \beta_{2} \beta_{4}\left(\Lambda-\mu I^{*}\right)} .
$$

Thus

$$
\begin{aligned}
X^{*} & =\frac{\mu^{2} k_{3}\left(d+\beta_{2} I^{*}\right)}{\beta_{1} \beta_{3}\left(\Lambda-\mu I^{*}\right)\left(d+\beta_{2} I^{*}\right)+\alpha \beta_{2} \beta_{4}\left(\Lambda-\mu I^{*}\right)}, \\
Z^{*} & =\frac{\mu^{2} \beta_{1} I^{*}\left(d+\beta_{2} I^{*}\right)}{\beta_{1} \beta_{3}\left(\Lambda-\mu I^{*}\right)\left(d+\beta_{2} I^{*}\right)+\alpha \beta_{2} \beta_{4}\left(\Lambda-\mu I^{*}\right)}, \\
U^{*} & =\frac{\alpha \mu^{2} \beta_{2} I^{*}}{\beta_{1} \beta_{3}\left(\Lambda-\mu I^{*}\right)\left(d+\beta_{2} I^{*}\right)+\alpha \beta_{2} \beta_{4}\left(\Lambda-\mu I^{*}\right)} .
\end{aligned}
$$

Substituting $X^{*}, Y^{*}, Z^{*}, U^{*}, S^{*}$ into (3.2), we obtain that the coordinates $I^{*}$ of an equilibrium solution will be the positive root of the quadratic equation

$$
h(I)=a I^{2}+b I+c=0,
$$

where

$$
a=\alpha_{1} K \mu \beta_{1} \beta_{2} \beta_{3}+\mu^{2} \beta_{1} \beta_{2} k_{4}>0,
$$




$$
\begin{aligned}
& b=\alpha_{1} K \beta_{1} \beta_{3}\left(d \mu-\Lambda \beta_{2}\right)+\alpha \alpha_{1} K \mu \beta_{2} \beta_{4}+\mu^{2} \beta_{2} k_{3} k_{5}+\mu^{2} \beta_{1} d k_{4}+\alpha \alpha_{1} \mu^{2} \beta_{2}, \\
& c=\mu^{2} k_{1} k_{2} k_{3}\left(1-R_{0}^{2}\right) .
\end{aligned}
$$

If the discriminant $\Delta=b^{2}-4 a c$ is positive, equation (3.8) has two real roots

$$
I_{1}=\frac{-b-\sqrt{\Delta}}{2 a}, \quad I_{2}=\frac{-b+\sqrt{\Delta}}{2 a} .
$$

Since all the state variables in model (2.1) are nonnegative, so $S^{*}\left(I^{*}\right)>0$ requires the coordinate $I^{*}$ to satisfy the inequality

$$
I^{*}<\frac{\Lambda}{\mu} .
$$

To completely determine the existence of the positive equilibrium of system (2.1), we must consider the following three possible cases:

(1) Assume $R_{0}>1$, then $c<0$ and thus $\Delta>0$. It is clear that $h(I)=0$ has a simple positive root, which we denote by $I_{2}$. In this case system (2.1) has a unique endemic equilibrium, which we denote by $E_{2}$.

(2) Suppose $R_{0}=1$, then $c=0$, and the equation $h(I)=0$ has two roots, which are 0 and $-\frac{b}{a}$. Hence system (2.1) has a unique endemic equilibrium, denoted by $E_{2}$, if and only if $b<0$.

(3) Suppose $R_{0}<1$, then $c>0$, and there are two possible subcases:

(i) If $b>0, h(I)=0$ does not have any positive root.

(ii) $h(I)=0$ has two positive roots $I_{1}$ and $I_{2}$ if and only if $\Delta>0$ and $b<0$; further, $h(I)=0$ has a double positive root $I^{*}$ if and only if $\Delta=0$ and $b<0$.

We can summarize the previous calculations in the following theorem.

Theorem 3.1 System (2.1) can have up to two positive equilibria. More precisely,

(1) if $R_{0}>1$, there exists a unique endemic equilibrium $E_{2}$;

(2) if $R_{0}=1$, there exists a unique endemic equilibrium $E_{2}$ if and only if $b<0$; otherwise, there is no endemic equilibrium;

(3) if $R_{0}<1$, and

(i) if $b>0$, there is no endemic equilibrium;

(ii) system (2.1) has two endemic equilibria $E_{1}$ and $E_{2}$ if and only if $\Delta>0$ and $b<0$; and these two equilibria coalesce into $E^{*}$ if and only if $\Delta=0$ and $b<0$; otherwise, there is no endemic equilibrium.

\subsection{Stability of the disease-free equilibrium point}

In this subsection, we show the stability of the disease-free equilibrium for model (2.1).

Theorem 3.2 For system (2.1), the disease-free equilibrium $E_{0}$ is locally asymptotically stable if $R_{0}<1$ and unstable if $R_{0}>1$. 
Proof The Jacobian matrix of system (2.1) about $E_{0}$ can be computed as

$$
J\left(E_{0}\right)=\left(\begin{array}{cccccc}
-k_{5} & -\alpha_{1} & -\alpha_{1} & -\alpha_{1} & 0 & -\frac{\beta_{1} \alpha_{1} K d}{k_{1} k_{2}} \\
\alpha & -d & 0 & 0 & 0 & -\frac{\beta_{2} \alpha \alpha_{1} K}{k_{1} k_{2}} \\
0 & 0 & -k_{3} & 0 & 0 & \frac{\beta_{1} \alpha_{1} K d}{k_{1} k_{2}} \\
0 & 0 & 0 & -k_{3} & 0 & \frac{\beta_{2} \alpha \alpha_{1} K}{k_{1} k_{2}} \\
0 & 0 & -\frac{\beta_{3} \Lambda}{\mu} & -\frac{\beta_{4} \Lambda}{\mu} & -\mu & 0 \\
0 & 0 & \frac{\beta_{3} \Lambda}{\mu} & \frac{\beta_{4} \Lambda}{\mu} & 0 & -\mu
\end{array}\right) .
$$

One eigenvalue of $J\left(E_{0}\right)$ is $-\mu$, which is negative, the others are the roots of the following quintic polynomial equation:

$$
\lambda^{5}+(A+C) \lambda^{4}+(D+A C+B) \lambda^{3}+(E+A D+B C) \lambda^{2}+(A E+B D) \lambda+B E=0
$$

where

$$
\begin{aligned}
& A=d+k_{5}>0, \\
& B=k_{1} k_{2}>0, \\
& C=k_{3}+k_{6}>0, \\
& D=k_{3} k_{6}+\mu k_{3}\left(1-R_{0}^{2}\right), \\
& E=\mu k_{3}^{2}\left(1-R_{0}^{2}\right), \\
& C D-E=k_{3} k_{6}\left[k_{3}+k_{6}+\mu\left(1-R_{0}^{2}\right)\right], \\
& A C-2 B=2 m\left(\alpha+\alpha_{1}\right)-2 \alpha \alpha_{1}+\mu \alpha+\mu \alpha_{1}+2 d\left(m+k_{6}\right), \\
& A^{2}-2 B=\alpha^{2}+\alpha_{1}^{2}+2 d \alpha+2 d \alpha_{1}+2 d^{2}>0 .
\end{aligned}
$$

Obviously, if $R_{0}<1$, then $D>0, E>0, C D-E>0$. In view of $0<\alpha \leq 1$ and $0<\alpha_{1} \leq 1$, we can get $A C-2 B>0$.

The Hertz determinants of the first to fifth order polynomial are as follows:

$$
\begin{aligned}
\Delta_{1}= & A+C>0, \\
\Delta_{2}= & A^{2} C+A B+A C^{2}+C D-E>0, \\
\Delta_{3}= & (C D-E)\left[A^{3}+A(A C-2 B)+E+A D\right]+A^{2} C^{2} B+A C B^{2}+A B C^{3}, \\
\Delta_{4}= & (C D-E)\left[A^{4} E+A B D\left(A^{2}-2 B\right)+A^{2} D E+A B D^{2}+A B^{3}+A^{2} B^{2} C\right. \\
& \left.+A^{2} E(A C-2 B)+A E^{2}+A B^{2} C^{2}+A^{2} B(C D-E)\right], \\
\Delta_{5}= & B E \Delta_{4} .
\end{aligned}
$$

If $R_{0}<1$, then we know $\Delta_{3}>0, \Delta_{4}>0, \Delta_{5}>0$. According to the criterion of RouthHurwitz, we can obtain that the disease-free equilibrium $E_{0}$ is locally asymptotically stable if and only if $R_{0}<1$. 
Theorem 3.3 For system (2.1), if $\hat{R}<1$, then the disease-free equilibrium $E_{0}$ is globally asymptotically stable, where

$$
\hat{R}=\sqrt{\frac{\beta_{2} \beta_{4} \alpha \alpha_{1} K \Lambda+\beta_{1} \beta_{3} \alpha_{1} K \Lambda d}{\mu^{2} d k_{3} k_{5}}} .
$$

Proof Obviously, $R_{0}<\hat{R}$. It follows from Theorem 3.2 that the disease-free equilibrium is locally asymptotically stable if $\hat{R}<1$. Thus we only show that it attracts all nonnegative solutions of model (2.1).

From the first, second, and fifth equations of system (2.1), we have

$$
\left\{\begin{array}{l}
\frac{d X}{d t} \leq \alpha_{1} K-\left(d+\alpha+\alpha_{1}\right) X \\
\frac{d Y}{d t} \leq \alpha X-d Y \\
\frac{d S}{d t} \leq \Lambda-\mu S
\end{array}\right.
$$

Thus we get that

$$
\limsup _{t \rightarrow \infty} X(t) \leq \frac{\alpha_{1} K}{k_{5}}, \quad \limsup _{t \rightarrow \infty} Y(t) \leq \frac{\alpha \alpha_{1} K}{d k_{5}}, \quad \limsup _{t \rightarrow \infty} S(t) \leq \frac{\Lambda}{\mu} .
$$

From the third, fourth, and sixth equations of system (2.1), we have

$$
\left\{\begin{array}{l}
\frac{d Z}{d t} \leq \frac{\beta_{1} \alpha_{1} K}{k_{5}} I-k_{3} Z \\
\frac{d U}{d t} \leq \frac{\beta_{2} \alpha \alpha_{1} K}{d k_{5}} I-k_{3} U, \\
\frac{d I}{d t} \leq \frac{\beta_{3} \Lambda}{\mu} Z+\frac{\beta_{4} \Lambda}{\mu} U-\mu I .
\end{array}\right.
$$

Consider the following comparison system:

$$
\left\{\begin{array}{l}
\frac{d y_{1}}{d t}=\frac{\beta_{1} \alpha_{1} K}{k_{5}} y_{3}-k_{3} y_{1}, \\
\frac{d y_{2}}{d t}=\frac{\beta_{2} \alpha \alpha_{1} K}{d k_{5}} y_{3}-k_{3} y_{2}, \\
\frac{d y_{3}}{d t}=\frac{\beta_{3} \Lambda}{\mu} y_{1}+\frac{\beta_{4} \Lambda}{\mu} y_{2}-\mu y_{3} .
\end{array}\right.
$$

System (3.9) is a linear and monotone system. The characteristic equation corresponding to system (3.9) is

$$
\left(\lambda+k_{3}\right)\left[\lambda^{2}+k_{6} \lambda+a_{1}\right]=0,
$$

where

$$
a_{1}=\frac{\mu^{2} d k_{3} k_{5}-\beta_{2} \beta_{4} \alpha \alpha_{1} K \Lambda-\beta_{1} \beta_{3} \alpha_{1} K \Lambda d}{\mu d k_{5}} .
$$

It is easy to know that real parts of all eigenvalues of (3.10) are negative provided that $\hat{R}<1$. So any solution which passes a nonnegative initial value of system (3.9) must satisfy the following equation:

$$
\lim _{t \rightarrow \infty} y_{i}(t)=0 \quad(i=1,2,3) .
$$


Let $0<Z(0)<y_{1}(0), 0<U(0)<y_{2}(0), 0<I(0)<y_{3}(0)$. If $\left(y_{1}(t), y_{2}(t), y_{3}(t)\right)$ is any solution of system (3.9) which passes a nonnegative initial value $\left(y_{1}(0), y_{2}(0), y_{3}(0)\right)$, according to the comparison principle of differential equations, we can obtain that $Z(t) \leq y_{1}(t), U(t) \leq$ $y_{2}(t), I(t) \leq y_{3}(t)$, for all $t \geq 0$.

Therefore, in view of Lemma 2.1, we have

$$
\lim _{t \rightarrow \infty} Z(t)=0, \quad \lim _{t \rightarrow \infty} U(t)=0, \quad \lim _{t \rightarrow \infty} I(t)=0 .
$$

The proof is completed.

\subsection{Existence of a backward bifurcation}

In most epidemiological models, the disease-free equilibrium loses its local stability through a forward bifurcation at $R_{0}=1$, at the same time a stable endemic equilibrium appears at this parameter value. This phenomenon can also be described as a supercritical transcritical bifurcation. However, under certain circumstances, such as nonlinear incidence, nonlinear recovery rate, and vector-borne transmission, a backward bifurcation may occur although $R_{0}<1$ (see [31]). The system exhibits an endemic equilibrium along with a stable disease-free equilibrium. There is then a subcritical transcritical bifurcation at $R_{0}=1$.

Now we take the vaccination rate of a citrus tree $\alpha$ as a bifurcation parameter. Solve for $\bar{\mu}$ from $R_{0}=1$, giving

$$
\bar{\mu}=\sqrt{\frac{\beta_{2} \beta_{4} \alpha \alpha_{1} K \Lambda+\beta_{1} \beta_{3} \alpha_{1} K \Lambda d}{k_{1} k_{2} k_{3}} .}
$$

We can use Theorem 4.1 in [32] to explore when system (2.1) undergoes either a forward or a backward bifurcation when $R_{0}=1$. So two quantities, labeled $\tilde{A}$ and $\tilde{B}$, need to be computed. In order to compute $\tilde{A}$ and $\tilde{B}$, a change of coordinates involving the right and left eigenvectors of the Jacobian matrix $J\left(E_{0}\right)$ associated with the eigenvalue $\lambda=0$ is required. We will express $\tilde{A}$ and $\tilde{B}$ in terms of parameters.

The Jacobian matrix $J\left(E_{0}\right)$ has a right eigenvector given by $w=\left(w_{1}, w_{2}, w_{3}, w_{4}, w_{5}, w_{6}\right)^{T}$, where

$$
\begin{aligned}
& w_{1}=\frac{m \alpha_{1} \beta_{2} Y_{0} w_{6}-\beta_{1} X_{0} d k_{4} w_{6}}{k_{1} k_{2} k_{3}}, \quad w_{2}=\frac{-\alpha \beta_{1} X_{0} k_{4} w_{6}-\beta_{2} Y_{0}\left(\alpha \alpha_{1}+k_{3} k_{5}\right) w_{6}}{k_{1} k_{2} k_{3}}, \\
& w_{3}=\frac{\beta_{1} X_{0} w_{6}}{k_{3}}, \quad w_{4}=\frac{\beta_{2} Y_{0} w_{6}}{k_{3}}, \quad w_{5}=-w_{6}, \quad w_{6}=w_{6} .
\end{aligned}
$$

It should be noted that $w_{2}<0$ and $w_{5}<0$. The Jacobian matrix $J\left(E_{0}\right)$ has a left eigenvector given by $v=\left(v_{1}, v_{2}, v_{3}, v_{4}, v_{5}, v_{6}\right)$, where

$$
v_{1}=v_{2}=v_{5}=0, \quad v_{3}=\frac{\beta_{3} S_{0} v_{6}}{k_{3}}, \quad v_{4}=\frac{\beta_{4} S_{0} v_{6}}{k_{3}}, \quad v_{6}=v_{6} .
$$

We can choose $w_{6}$ and $v_{6}$ satisfy

$$
v_{6} \cdot w_{6}=\left(\frac{\beta_{2} \beta_{4} \alpha \alpha_{1} K \Lambda+\beta_{1} \beta_{3} \alpha_{1} K \Lambda d}{\mu k_{1} k_{2} k_{3}^{2}}+1\right)^{-1}>0
$$

such that $v \cdot w=1$. 
In order to follow the notations introduced in the literature [32], we set $x_{1}=X-\frac{\alpha_{1} K d}{k_{1} k_{2}}$, $x_{2}=Y-\frac{\alpha \alpha_{1} K}{k_{1} k_{2}}, x_{3}=Z, x_{4}=U, x_{5}=S-\frac{\Lambda}{\mu}, x_{6}=I, W=\left(x_{1}, x_{2}, x_{3}, x_{4}, x_{5}, x_{6}\right)^{T}, \phi=\mu-\bar{\mu}$. Thus system (2.1) can be rewritten as follows:

$$
\frac{d W}{d t}=F=\left(f_{1}, f_{2}, f_{3}, f_{4}, f_{5}, f_{6}\right)^{T}
$$

By direct computation we can get the associated non-zero partial derivatives of $F$ at the disease-free equilibrium:

$$
\begin{aligned}
& \frac{\partial^{2} f_{1}}{\partial x_{1} \partial x_{6}}(\mathbf{0}, 0)=-\beta_{1}, \quad \frac{\partial^{2} f_{2}}{\partial x_{2} \partial x_{6}}(\mathbf{0}, 0)=-\beta_{2}, \quad \frac{\partial^{2} f_{3}}{\partial x_{1} \partial x_{6}}(\mathbf{0}, 0)=\beta_{1}, \\
& \frac{\partial^{2} f_{4}}{\partial x_{2} \partial x_{6}}(\mathbf{0}, 0)=\beta_{2}, \quad \frac{\partial^{2} f_{5}}{\partial x_{3} \partial x_{5}}(\mathbf{0}, 0)=-\beta_{3}, \quad \frac{\partial^{2} f_{5}}{\partial x_{4} \partial x_{5}}(\mathbf{0}, 0)=-\beta_{4}, \\
& \frac{\partial^{2} f_{6}}{\partial x_{3} \partial x_{5}}(\mathbf{0}, 0)=\beta_{3}, \quad \frac{\partial^{2} f_{6}}{\partial x_{4} \partial x_{5}}(\mathbf{0}, 0)=-\beta_{4},
\end{aligned}
$$

and all other derivatives are equal to zero.

Consequently, we can compute the two quantities $\tilde{A}, \tilde{B}$.

$$
\tilde{A}=\sum v_{k} w_{i} w_{j} \frac{\partial^{2} f_{k}}{\partial x_{i} \partial x_{j}}(\mathbf{0}, 0)=\frac{-g(\alpha)}{k_{3}^{2} k_{1} k_{2}} v_{6} w_{6}^{2},
$$

where

$$
\begin{aligned}
& g(\alpha)=A_{1} \alpha^{2}+A_{2} \alpha+A_{3}, \\
& A_{1}=\beta_{2} \beta_{4}\left[\beta_{2} \Lambda k_{4}+\mu k_{2} k_{3}\right]>0, \\
& A_{2}=\beta_{1} \beta_{2} \beta_{4} \Lambda d k_{4}+\beta_{2} \beta_{4} k_{2} k_{3}\left(\beta_{2} \Lambda+\mu d\right)+\mu d \beta_{1} \beta_{3} k_{2} k_{3}-m \alpha_{1} \beta_{1} \beta_{2} \beta_{3} \Lambda, \\
& A_{3}=\beta_{1}^{2} \beta_{3} \Lambda d^{2} k_{4}+\beta_{1} \beta_{3} \mu d^{2} k_{2} k_{3}>0 .
\end{aligned}
$$

Denote $\Delta^{\prime}$ is discriminant of $g(\alpha)$. Then $g(\alpha)=0$ have two positive real roots $\alpha^{*}=\frac{-A_{2}-\sqrt{\Delta^{\prime}}}{2 A_{1}}$ and $\alpha^{* *}=\frac{-A_{2}+\sqrt{\Delta^{\prime}}}{2 A_{1}}$ if and only if $\Delta^{\prime}>0$ and $A_{2}<0$.

Note that $\frac{\partial^{2} f_{5}}{\partial x_{5} \partial \phi}(\mathbf{0}, 0)=\frac{\partial^{2} f_{6}}{\partial x_{6} \partial \phi}(\mathbf{0}, 0)=1$, and all other derivatives $\frac{\partial^{2} f_{k}}{\partial x_{i} \partial \phi}(\mathbf{0}, 0)$ are equal to zero. So we can calculate $\tilde{B}$ by substituting the vectors $v$ and $w$ and the respective partial derivatives into the expression

$$
\tilde{B}=\sum v_{k} w_{i} \frac{\partial^{2} f_{k}}{\partial x_{i} \partial \phi}(\mathbf{0}, 0)=w_{6} v_{6}>0
$$

According to the above discussion, we can conclude that $\tilde{A}>0$ and $\tilde{B}>0$ if and only if $\Delta^{\prime}>0, A_{2}<0$, and $\alpha^{*}<\alpha<\alpha^{* *}$, which is the defining condition for a backward bifurcation [32].

Theorem 3.4 System (2.1) undergoes, at $R_{0}=1$, a backward bifurcation if and only if $\Delta^{\prime}>0, A_{2}<0$, and $\alpha^{*}<\alpha<\alpha^{* *}$; otherwise, system (2.1) undergoes a forward bifurcation. 
Remark 3.1 In the case of a backward bifurcation, there exists a saddle-node bifurcation point $R_{0}^{c}$ such that for $0<R_{0}<R_{0}^{c}$, a unique stable disease-free equilibrium point exists, and, for $R_{0}^{c}<R_{0}<1$, the stable disease-free equilibrium point coexists with two endemic points. We can determine $R_{0}^{c}$ by setting $\Delta=0$, and get

$$
R_{0}^{c}=\sqrt{1-\frac{b^{2}}{4 a \mu^{2} k_{1} k_{2} k_{3}}} .
$$

Remark 3.2 From Remark 3.1, we know that the backward bifurcation gives a further sub-threshold condition beyond the reproduction number for the control of HLB, i.e., $R_{0}<R_{0}^{c}<1$. The existence of the backward bifurcation illustrates that the long term HLB activity in a citrus orchard depends on the initial population sizes of citrus trees and psyllids.

\section{Permanence}

In this section, we demonstrate the permanence of system (2.1). We first give some notations and a lemma.

Let $\tilde{K}$ be a matrix space, $f: \tilde{K} \rightarrow \tilde{K}$ be a continuous map, and $K_{0} \subseteq \tilde{K}$ be an open set.

Define

$$
\partial K_{0}=\tilde{K} \backslash K_{0}, \quad \text { and } \quad M_{\partial}=\left\{x \in \partial K_{0}: f^{n}(x) \in \partial K_{0}, \forall n \in N\right\} .
$$

$A_{\partial}$ is a maximal compact invariant set of $f$ in $\partial K_{0}$. A finite sequence $\left\{M_{1}, \ldots, M_{k}\right\}$ are disjoint, compact, and invariant subsets of $\partial K_{0}$, and each of them is isolated in $\partial K_{0}$.

Lemma 4.1 (See [33]) Assume that

(I) $f\left(K_{0}\right) \subset K_{0}$ and $f$ has a global attractor $A$.

(II) The maximal compact invariant set $A_{\partial}=A \bigcap M_{\partial}$ off in $\partial K_{0}$, possibly empty, has an acyclic covering $\tilde{M}=\left\{M_{1}, \ldots, M_{k}\right\}$ with the following properties:

(a) $M_{i}$ is isolated in $\tilde{K}$.

(b) $W^{s}\left(M_{i}\right) \bigcap K_{0}=\emptyset$ for each $1 \leq i \leq k$.

Then $f$ is uniformly persistent with respect to $\left(K_{0}, \partial K_{0}\right)$, i.e., there is $\eta>0$ such that for any compact internally chain transitive set $L$ with $L \nsubseteq\left\{M_{i}\right.$, for all $\left.1 \leq i \leq k\right\}, \inf _{x \in L} d(x$, $\left.\partial K_{0}\right)>\eta$.

Theorem 4.1 If $R_{0}>1$, system (2.1) is permanent.

Proof Since $R_{0}>1$, then we can choose sufficiently small $\varepsilon>0$ such that

$$
\Upsilon \triangleq \frac{\gamma_{1}+\gamma_{2}}{\mu k_{2} k_{3}\left(d+\beta_{2} \varepsilon\right)\left(d+\alpha+\beta_{1} \varepsilon\right)\left(\beta_{3} \varepsilon+\beta_{4} \varepsilon+\mu\right)}>1,
$$

where

$$
\begin{aligned}
& \gamma_{1}=\beta_{1} \beta_{3}\left(\Lambda-\varepsilon\left(\beta_{3} \varepsilon+\beta_{4} \varepsilon+\mu\right)\right)\left(d+\beta_{2} \varepsilon\right)\left(\alpha_{1} K d-k_{2} \varepsilon\left(d+\alpha+\beta_{1} \varepsilon\right)\right), \\
& \gamma_{2}=\beta_{2} \beta_{4}\left(\Lambda-\varepsilon\left(\beta_{3} \varepsilon+\beta_{4} \varepsilon+\mu\right)\right)\left(\alpha \alpha_{1} K d-k_{2} \varepsilon\left(d+\beta_{2} \varepsilon\right)\left(d+\alpha+\beta_{1} \varepsilon\right)\right) .
\end{aligned}
$$


To apply Lemma 4.1, for system (2.1), we define $\tilde{K}=\left\{(X, Y, Z, U, S, I) \in R_{+}^{6}\right\}, K_{0}=$ $\{(X, Y, Z, U, S, I) \in \tilde{K}: X \geq 0, Y \geq 0, Z>0, U>0, S \geq 0, I>0\}$, and $\partial K_{0}=\tilde{K} \backslash K_{0}$, and denote $u\left(t, x^{0}\right)$ as the unique solution of system (2.1) with the initial value $x^{0}=\left(X^{0}, Y^{0}, Z^{0}, U^{0}\right.$, $\left.S^{0}, I^{0}\right)$.

Define the Poincaré map $P: \tilde{K} \rightarrow \tilde{K}$ associated with system (2.1) as follows:

$$
P\left(x^{0}\right)=u\left(1, x^{0}\right), \quad \forall x^{0} \in \tilde{K} .
$$

It is easy to see that both $\tilde{K}$ and $K_{0}$ are positively invariant and $P$ is point dissipative.

Set

$$
M_{\partial}=\left\{\left(X^{0}, Y^{0}, Z^{0}, U^{0}, S^{0}, I^{0}\right) \in \partial K_{0} \mid P^{m}\left(X^{0}, Y^{0}, Z^{0}, U^{0}, S^{0}, I^{0}\right) \in \partial K_{0}, \forall m \in N\right\} .
$$

We claim that

$$
M_{\partial}=\{(X, Y, 0,0, S, 0) \mid X \geq 0, Y \geq 0, S \geq 0\} .
$$

Obviously, $\{(X, Y, 0,0, S, 0) \mid X \geq 0, Y \geq 0, S \geq 0\} \subseteq M_{\partial}$. Next we want to show

$$
M_{\partial} \backslash\{(X, Y, 0,0, S, 0) \mid X \geq 0, Y \geq 0, S \geq 0\}=\emptyset .
$$

If it does not hold, then there exists a point $\left(X^{0}, Y^{0}, Z^{0}, U^{0}, S^{0}, I^{0}\right) \in M_{\partial} \backslash\{(X, Y, 0,0, S, 0) \mid$ $X \geq 0, Y \geq 0, S \geq 0\}$.

Case (i). $Z^{0}=0, U^{0}>0, I^{0}>0$. It is obvious that $X(t)>0, I(t)>0$ for any $t>0$. Then, from the third equation of system (2.1), $\left.\frac{d Z}{d t}\right|_{t=0}=\beta_{1} X(0) I(0)>0$ holds. It follows that $(X, Y, Z, U, S, I) \notin \partial K_{0}$ for $0<t \ll 1$.This is a contradiction.

Similarly, we can prove the other cases: (a) $Z^{0}>0, U^{0}=0, I^{0}>0$, and (b) $Z^{0}>0, U^{0}>0$, $I^{0}=0$.

Case (ii). $Z^{0}=U^{0}=0, I^{0}>0$. It is obvious that $X(t)>0, Y(t)>0, I(t)>0$ for any $t>0$. Then, from the third, fourth equations of system (2.1), we get $\left.\frac{d Z}{d t}\right|_{t=0}=\beta_{1} X(0) I(0)>0$, $\left.\frac{d U}{d t}\right|_{t=0}=\beta_{2} Y(0) I(0)>0$ hold. It follows that $(X, Y, Z, U, S, I) \notin \partial K_{0}$ for $0<t \ll 1$. This is a contradiction.

Similarly, we can prove the other cases: (c) $Z^{0}=I^{0}=0, U^{0}>0$, and (d) $U^{0}=I^{0}=0$, $Z^{0}>0$.

That is to say, for any $\left(X^{0}, Y^{0}, Z^{0}, U^{0}, S^{0}, I^{0}\right) \notin\{(X, Y, 0,0, S, 0) \mid X \geq 0, Y \geq 0, S \geq 0\}$, then $\left(X^{0}, Y^{0}, Z^{0}, U^{0}, S^{0}, I^{0}\right) \notin M_{\partial}$. Therefore we have

$$
M_{\partial}=\{(X, Y, 0,0, S, 0) \mid X \geq 0, Y \geq 0, S \geq 0\} .
$$

In the following, we need to prove

$$
W^{s}\left(E_{0}\right) \cap K_{0}=\emptyset .
$$

We write $x^{0}=\left(X^{0}, Y^{0}, Z^{0}, U^{0}, S^{0}, I^{0}\right) \in K_{0}$. By the continuity of the solutions with respect to the initial conditions, we know that, for any given $\varepsilon>0$, there exists $\delta_{0}>0$ such that for all $x^{0} \in K_{0}$ with $\left|x^{0}-E_{0}\right| \leq \delta_{0}$, it holds that

$$
\left\|u\left(t, x^{0}\right)-u\left(t, E_{0}\right)\right\| \leq \varepsilon, \quad \forall t \in[0,1] .
$$


Now we proceed by contradiction to prove that

$$
\limsup _{t \rightarrow \infty} d\left(P^{m}\left(x^{0}\right), E_{0}\right) \geq \delta_{0}
$$

If (4.2) does not hold, then

$$
\limsup _{t \rightarrow \infty} d\left(P^{m}\left(x^{0}\right), E_{0}\right)<\delta_{0},
$$

for some $x^{0} \in K_{0}$.

Without loss of generality, we suppose that

$$
d\left(P^{m}\left(x^{0}\right), E_{0}\right)<\delta_{0}, \quad \forall m \in N
$$

By the continuity of the solutions with respect to the initial values, we obtain

$$
\left\|u\left(t, P^{m}\left(x^{0}\right)\right)-u\left(t, E_{0}\right)\right\| \leq \varepsilon, \quad \forall t \in[0,1], \forall m \in N .
$$

For any $\tilde{t}_{1} \geq 0$, there exists an integer $m \geq 0$ such that $\tilde{t}_{1}=m+t^{\prime}$, where $t^{\prime} \in[0,1]$.

Then we have

$$
\left\|u\left(\tilde{t}_{1}, x^{0}\right)-u\left(\tilde{t}_{1}, E_{0}\right)\right\|=\left\|u\left(t^{\prime}, P^{m}\left(x^{0}\right)\right)-u\left(t^{\prime}, E_{0}\right)\right\| \leq \varepsilon,
$$

for any $\tilde{t}_{1} \geq 0$, which implies that

$$
0 \leq Z(t) \leq \varepsilon, \quad 0 \leq U(t) \leq \varepsilon, \quad 0 \leq I(t) \leq \varepsilon .
$$

From (2.1) and (4.3), we have

$$
\left\{\begin{array}{l}
\frac{d X}{d t} \geq \alpha_{1} K-\frac{\alpha_{1}^{2} K}{k_{2}}-\left(d+\alpha+\beta_{1} \varepsilon\right) X \\
\frac{d Y}{d t} \geq \alpha X-\left(d+\beta_{2} \varepsilon\right) Y \\
\frac{d S}{d t} \geq \Lambda-\left(\beta_{3} \varepsilon+\beta_{4} \varepsilon+\mu\right) S
\end{array}\right.
$$

Consequently, we can easily obtain that there exists $T>0$ such that for all $t>T$

$$
\begin{aligned}
& X(t) \geq \frac{\alpha_{1} K d}{k_{2}\left(d+\alpha+\beta_{1} \varepsilon\right)}-\varepsilon, \quad Y(t) \geq \frac{\alpha \alpha_{1} K d}{k_{2}\left(d+\alpha+\beta_{1} \varepsilon\right)\left(d+\beta_{2} \varepsilon\right)}-\varepsilon, \\
& S(t) \geq \frac{\Lambda}{\beta_{3} \varepsilon+\beta_{4} \varepsilon+\mu}-\varepsilon .
\end{aligned}
$$

Substituting (4.4) into the third, fourth, and sixth equations of system (2.1), we have that for $t>T$

$$
\left\{\begin{array}{l}
\frac{d Z}{d t} \geq \beta_{1} I\left(\frac{\alpha_{1} K d}{k_{2}\left(d+\alpha+\beta_{1} \varepsilon\right)}-\varepsilon\right)-k_{3} Z, \\
\frac{d U}{d t} \geq \beta_{2} I\left(\frac{\alpha \alpha_{1} K d}{k_{2}\left(d+\alpha+\beta_{1} \varepsilon\left(d+\beta_{2} \varepsilon\right)\right.}-\varepsilon\right)-k_{3} U, \\
\frac{d I}{d t} \geq \beta_{3} Z\left(\frac{\Lambda}{\beta_{3} \varepsilon+\beta_{4} \varepsilon+\mu}-\varepsilon\right)+\beta_{4} U\left(\frac{\Lambda}{\beta_{3} \varepsilon+\beta_{4} \varepsilon+\mu}-\varepsilon\right)-\mu I .
\end{array}\right.
$$


Consider the following comparison system:

$$
\left\{\begin{array}{l}
\frac{d \tilde{Z}}{d t}=\beta_{1} \tilde{I}\left(\frac{\alpha_{1} K d}{k_{2}\left(d+\alpha+\beta_{1} \varepsilon\right)}-\varepsilon\right)-k_{3} \tilde{Z} \\
\frac{d \tilde{U}}{d t}=\beta_{2} \tilde{I}\left(\frac{\alpha \alpha_{1} K d}{k_{2}\left(d+\alpha+\beta_{1} \varepsilon\right)\left(d+\beta_{2} \varepsilon\right)}-\varepsilon\right)-k_{3} \tilde{U} \\
\frac{d \tilde{I}}{d t}=\beta_{3} \tilde{Z}\left(\frac{\Lambda}{\beta_{3} \varepsilon+\beta_{4} \varepsilon+\mu}-\varepsilon\right)+\beta_{4} \tilde{U}\left(\frac{\Lambda}{\beta_{3} \varepsilon+\beta_{4} \varepsilon+\mu}-\varepsilon\right)-\mu \tilde{I}
\end{array}\right.
$$

Denote the coefficient matrix of system (4.6) by $H$, here

$$
H=\left(\begin{array}{ccc}
-k_{3} & 0 & \beta_{1}\left(\frac{\alpha_{1} K d}{k_{2}\left(d+\alpha+\beta_{1} \varepsilon\right)}-\varepsilon\right) \\
0 & -k_{3} & \beta_{2}\left(\frac{\alpha \alpha_{1} K d}{k_{2}\left(d+\alpha+\beta_{1} \varepsilon\right)\left(d+\beta_{2} \varepsilon\right)}-\varepsilon\right) \\
\beta_{3}\left(\frac{\Lambda}{\beta_{3} \varepsilon+\beta_{4} \varepsilon+\mu}-\varepsilon\right) & \beta_{4}\left(\frac{\Lambda}{\beta_{3} \varepsilon+\beta_{4} \varepsilon+\mu}-\varepsilon\right) & -\mu
\end{array}\right) .
$$

Thus, we get the characteristic equation of $H$ :

$$
\left(\lambda+k_{3}\right)\left(\lambda^{2}+k_{6} \lambda+c\right)=0
$$

where

$$
k_{6}=d+m+\mu>0, \quad c=\mu k_{2} k_{3}\left(d+\beta_{2} \varepsilon\right)\left(d+\alpha+\beta_{1} \varepsilon\right)\left(\beta_{3} \varepsilon+\beta_{4} \varepsilon+\mu\right)(1-\Upsilon) .
$$

It follows from (4.1) that $c<0$ provided that $R_{0}>1$. Then there exists a unique positive root $\lambda^{*}$ of characteristic polynomial of $H$. By [34], there exists a positive characteristic vector denoted by $\left(\tilde{Z}_{1}, \tilde{U}_{1}, \tilde{I}_{1}\right)$ such that $e^{\lambda^{*} t}\left(\tilde{Z}_{1}, \tilde{U}_{1}, \tilde{I}_{1}\right)$ is a solution of system (4.6). Further, for any given initial values $(Z(0), U(0), I(0))$, there exists sufficiently small positive $q$ such that $q \tilde{Z}_{1}<Z(0), q \tilde{U}_{1}<U(0), q \tilde{I}_{1}<I(0)$. Any solution $(\tilde{Z}(t), \tilde{U}(t), \tilde{I}(t))$ of system (4.6) which passes initial value $\tilde{Z}(0)=q \tilde{Z}_{1}, \tilde{U}(0)=q \tilde{U}_{1}, \tilde{I}(0)=q \tilde{I}_{1}$ must satisfy $\tilde{Z}(t) \rightarrow \infty, \tilde{U}(t) \rightarrow \infty$, $\tilde{I}(t) \rightarrow \infty$, as $t \rightarrow \infty$. According to the comparison principle in differential equations, the solution $(Z(t), U(t), I(t))$ of system (4.5) with initial values $(Z(0), U(0), I(0))$ must satisfy $Z(t)>\tilde{Z}(t), U(t)>\tilde{U}(t), I(t)>\tilde{I}(t)$. Therefore, $Z(t) \rightarrow \infty, U(t) \rightarrow \infty, I(t) \rightarrow \infty$, as $t \rightarrow \infty$. This is a contradiction. Thus, we have proved that (4.2) holds and $P$ is weakly uniformly persistent with respect to $\left(K_{0}, \partial K_{0}\right)$.

We can easily obtain that $P$ has a global attractor $E_{0}$. It is easy to obtain that $E_{0}$ is an isolated invariant set in $\tilde{K}$ and $W^{s}\left(E_{0}\right) \cap K_{0}=\emptyset$. We know that $E_{0}$ is acyclic in $M_{\partial}$, and every solution in $M_{\partial}$ converges to $E_{0}$. According to Zhao [33], we have that $P$ is uniformly persistent with respect to $\left(K_{0}, \partial K_{0}\right)$. This implies that the solution of system (2.1) is uniformly persistent with respect to $\left(K_{0}, \partial K_{0}\right)$. This completes the proof.

\section{Numerical simulation}

In this section, we first provide results from numerical simulations of model (2.1) that demonstrate and support our theoretical results. The model will be applied to study the transmission of HLB in the South of Jiangxi, China. We need to estimate the model parameters in order to carry out the numerical simulations. The values of parameters of model (2.1) are given in Table 1. We explain the parameter values as follows:

- The average life expectancy of trees was from 20 years to 30 years [35]. We can take 25 as the current average life expectancy. Thus, the natural death rate of citrus trees $d=\frac{1}{25}=0.04$. 
Table 1 Parameter values used for numerical simulations of the HLB model

\begin{tabular}{llll}
\hline Parameters & Values & Unit & References \\
\hline$d$ & 0.04 & year $^{-1}$ & {$[35]$} \\
$\mu$ & 5.7394 & year $^{-1}$ & {$[36]$} \\
$K$ & 2,000 & - & {$[36]$} \\
$\alpha_{1}$ & year $^{-1}$ & Estimation \\
$\beta_{1}$ & 0.6 & year $^{-1}$ & {$[36]$} \\
$\beta_{2}$ & 0.000243333 & year $^{-1}$ & Estimation \\
$\beta_{3}$ & 0.00000365 & year $^{-1}$ & {$[36]$} \\
$\beta_{4}$ & 0.000194667 & year $^{-1}$ & Estimation \\
$\Lambda$ & 0.000214133 & year $^{-1}$ & {$[36]$} \\
$\alpha$ & $6,028,433$ & year $^{-1}$ & - \\
$m$ & $0 \sim 1$ & year $^{-1}$ & - \\
\hline
\end{tabular}

- The annual average temperature of Jiangxi Province is from 8.6 to 20.6 degree centigrade in 2010 . So we can choose the average temperature $T=17^{\circ} \mathrm{C}$.

- It follows from the literature [36-38] that the natural mortality of psyllids is taken as the form $\mu=\frac{1}{L} 365$, in which $L=-0.14221 * T^{2}+4.31998 * T+31.25498$. Thus, we can get $\mu=5.7394$.

- The maximum number of trees that can be planted in the grove is 2,000, i.e., $K=2,000$. According to the implementation of control measures for HLB in the South of Jiangxi, we take the replanting rate $\alpha_{1}=0.6$.

- Using the same transmission forms in [36], we get $\beta_{1}=\frac{0.45625\left(d+\alpha_{1}\right)}{\alpha_{1} K}=0.000243333$, $\beta_{3}=\frac{0.365\left(d+\alpha_{1}\right)}{\alpha_{1} K}=0.000194667$. Further, we take $\beta_{2}=0.015 \beta_{1}$ and $\beta_{4}=1.1 \beta_{3}$, then $\beta_{2}=0.00000365$ and $\beta_{4}=0.000214133$.

- From literature [36] and [37], we know the recruitment rate of psyllid $\Lambda=\frac{3 \alpha_{1} K * E F D * P_{e a} * M D R}{\mu\left(d+\alpha_{1}\right)}$, where $E F D=0.0107 * 365 * T *(T-13) * \sqrt{30.8-T}$, $P_{\text {ea }}=0.47192+0.0109 * T, M D R=5.286 * 10^{-5} * 365 * T *(T-10.02) * \sqrt{34.17-T}$, then we can calculate $\Lambda=6,028,433$.

All citrus trees infected by HLB must be removed in the South of Jiangxi. But HLB has an incubation period during which the infected trees do not show symptoms, so we take the removing rate $m=0.6$ by empirical estimation. If we choose the vaccination rate $\alpha=0.1$, by (3.1), we can obtain the basic reproductive number for the South of Jiangxi $R_{0}=2.75$. Sensitivity analysis of all constant parameters that are included in $R_{0}$ is performed (see Figure 2). For the parameters, including $\Lambda, \mu, K, m, \beta_{4}, \beta_{3}, \beta_{2}, \beta_{1}, \alpha, d, \alpha_{1}$, we vary their value by $10 \%$ and plot the effects on $R_{0}$. From Figure 2 , we can clearly observe that the natural mortality of psyllid population $(\mu)$ has the most effects on $R_{0}$ of all the constant parameters. The reason is the parameter is involved in both directions of transmission: from a tree to a psyllid and vice versa. Note that parameters $\alpha, m, \mu$ have the opposite effects on $R_{0}$. For example, an increase in $\alpha$ decreases $R_{0}$, whereas other parameters are positively correlated with $R_{0}$. Apart from $\alpha_{1}$ (invariability), parameters $\beta_{1}, \beta_{3}, K$ have the effect on $R_{0}$. By increasing the rate of vaccinating protecting virus $\alpha$, the roguing rate $m$, the mortality of psyllid population $\mu$, it is possible to reduce the prevalence of the disease.

In addition, from Figure 2 we can find that the replanting rate $\left(\alpha_{1}\right)$ is less sensitive to $R_{0}$, but the different values of $\alpha_{1}$ can affect the peak values of the infected trees and the times to reach peak value. Figure 3 reflects that, along with the increase in value $\alpha_{1}$, the peak value delays in time and increases in value. Therefore, the risk of HLB spread would reduce with the decrease of replanting magnitude. 


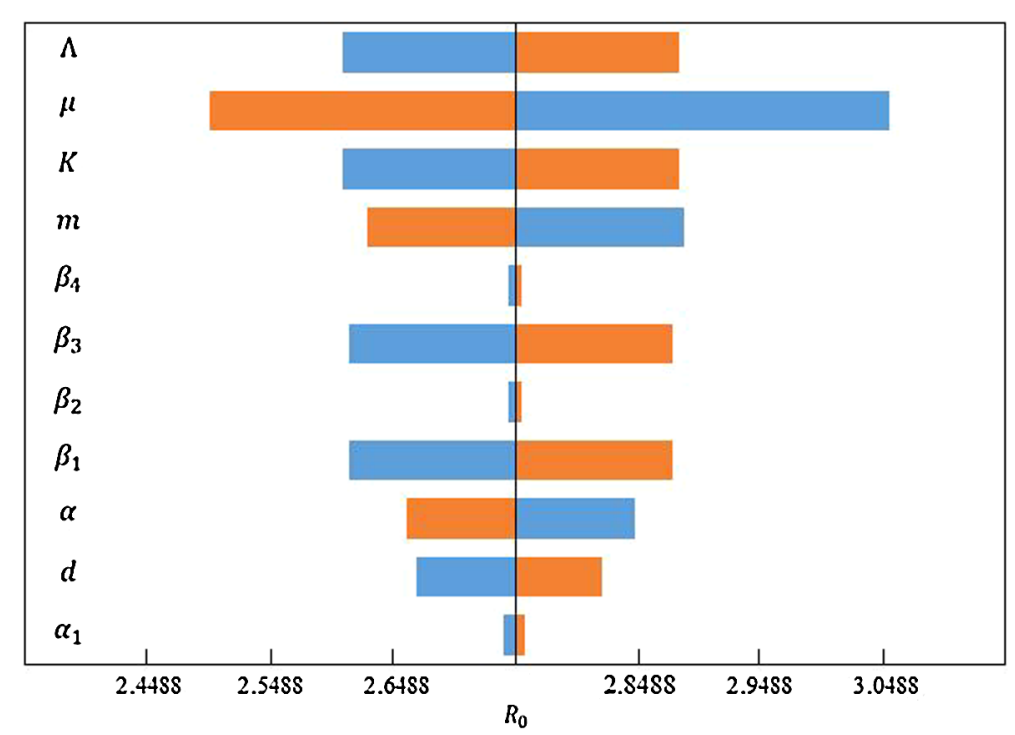

Figure 2 The sensitivity of $\boldsymbol{R}_{\mathbf{0}}$ to changes in constant parameters. Each parameter is varied by $10 \%$ to assess the impact on $R_{0}$. Decreasing each parameter by $10 \%$ (e.g., $0.9 \alpha$ ) is indicated in blue; increasing each parameter by $10 \%$ (e.g., $1.1 \alpha$ ) is indicated in orange.

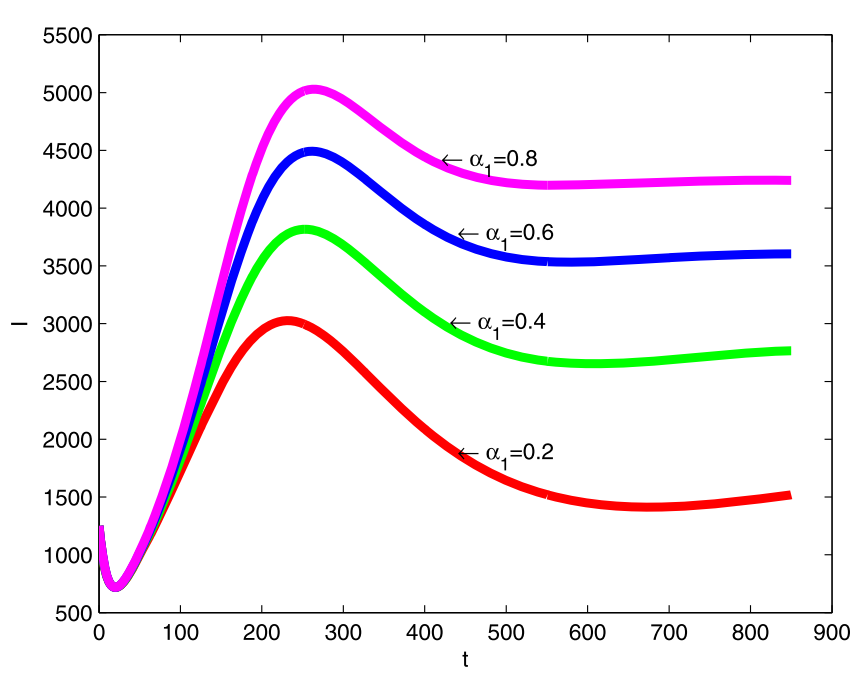

Figure 3 Time series plot of infectious tree population with different replanting rates.

Figure 4 shows the dependence of the basic reproductive number $R_{0}$ on two control strengths of vaccination and removing the infected tree. $R_{0}$ decreases as $m$ and $\alpha$ increase from 0 to 1 , and it is more sensitive when $m$ and $\alpha$ are small. It implies that proper cross protection or removing infected trees can effectively control the spread of HLB.

Next, in order to show the effects of cross protection on the total infective population, we fix the vaccination rate $\alpha=0,0.4,0.8$, respectively. The numerical results (see Figure 5) show that there is high infected value without cross protection $(\alpha=0)$; however, there are low density values after inoculating the protecting virus strains. It shows that the protecting virus without causing undue harm plays a key role in HLB virus control in plants. It appears to offer a promising measure for HLB control. 


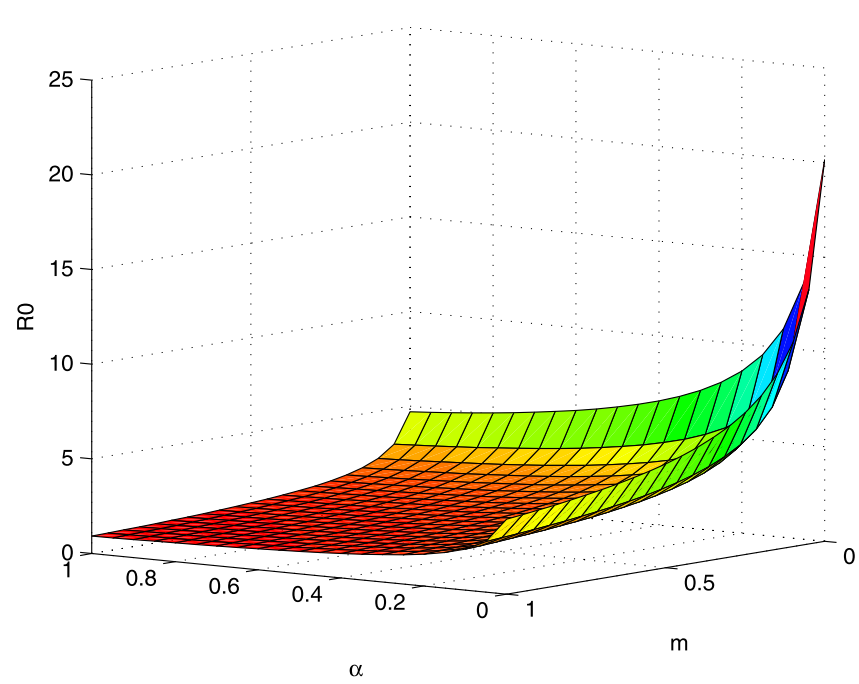

Figure 4 The dependence of the basic reproductive number on the strength of vaccination and removing infected tree.

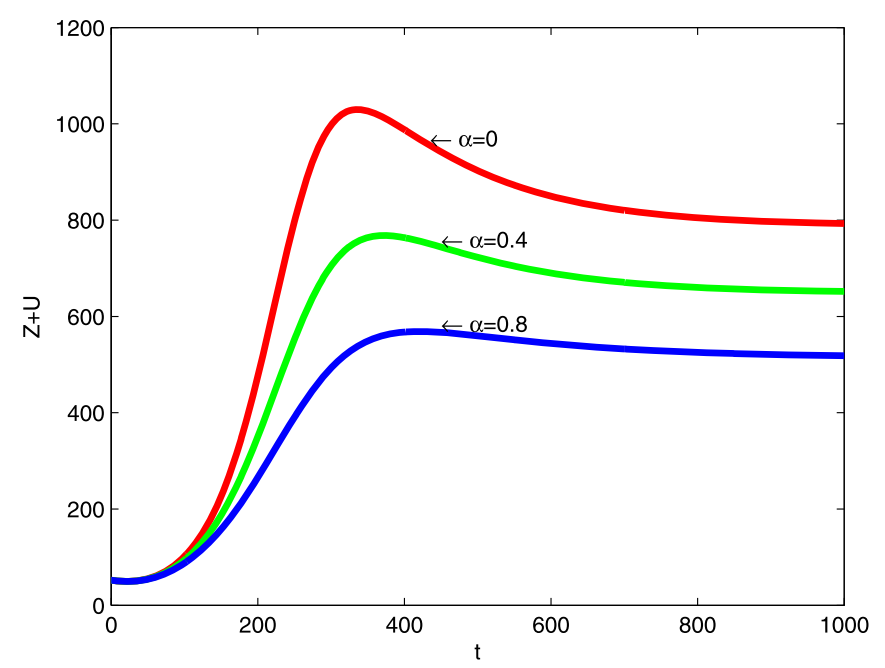

Figure 5 Time series plot of the total infectious tree population with different vaccination rates.

Finally, numerical simulations are carried out to illustrate the effectiveness of the obtained results. For the simulations that follow, we applied this set of parameters shown in Table 1 unless otherwise stated.

Set $m=0.988$. According to the result of Theorem 3.4, we know that if $\alpha \in[0.81,0.86]$, a backward bifurcation can occur, that is, for $R_{0}<1$, a unique disease-free equilibrium and two subcritical endemic equilibria coexist, but for $R_{0}>1$, a unique endemic equilibrium exists (see Figure 6(a)). Moreover, if $\alpha \in[0,0.81) \cup(0.86,1]$, a forward bifurcation occurs, that is, for $R_{0}<1$, a unique disease-free equilibrium exists, which is stable, but its stability is lost at $R_{0}=1$, in which a stable endemic equilibrium arises by a transcritical bifurcation (see Figure 6(b)). 


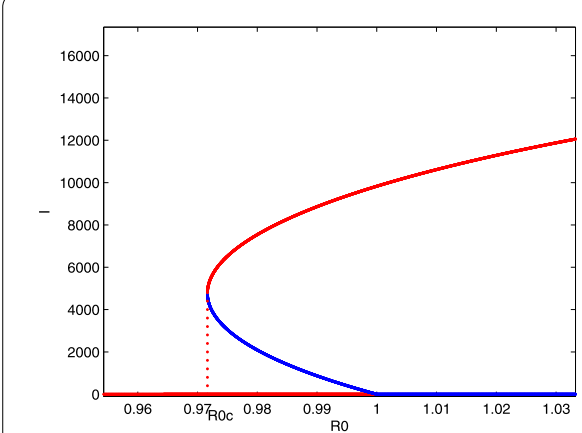

(a)

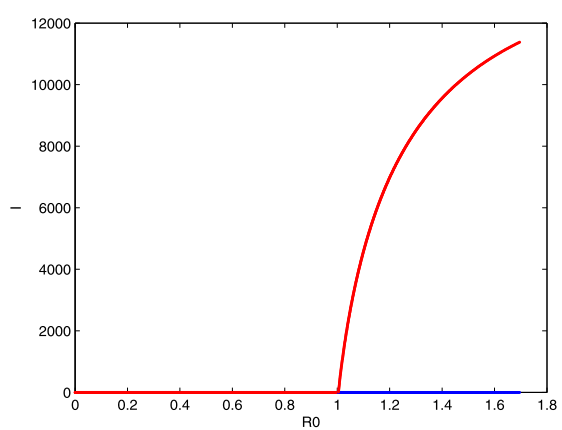

(b)

Figure 6 Bifurcation diagram in $\left(\boldsymbol{R}_{0}, \boldsymbol{l}\right)$ plane. (a) Backward bifurcation $(0.81 \leq \alpha \leq 0.86)$. (b) Forward bifurcation $(0.2 \leq \alpha \leq 0.7)$.

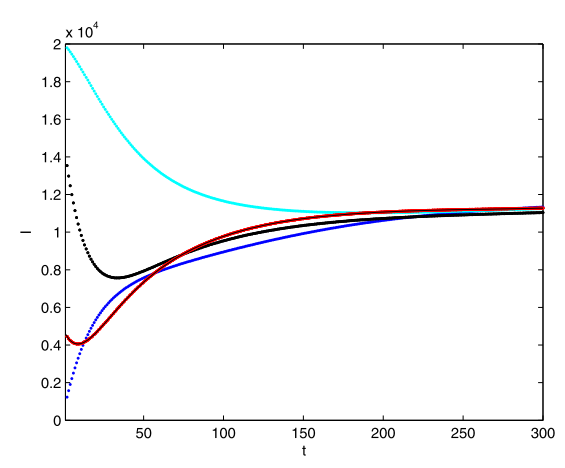

(a) $R_{0}=1.1886, \alpha=0.5$

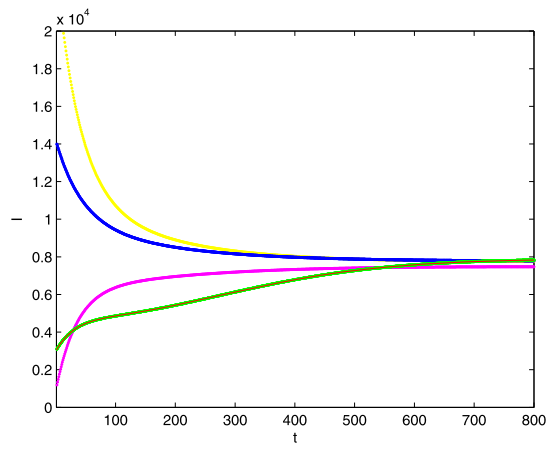

(c) $R_{0}=0.9797, \alpha=0.85$

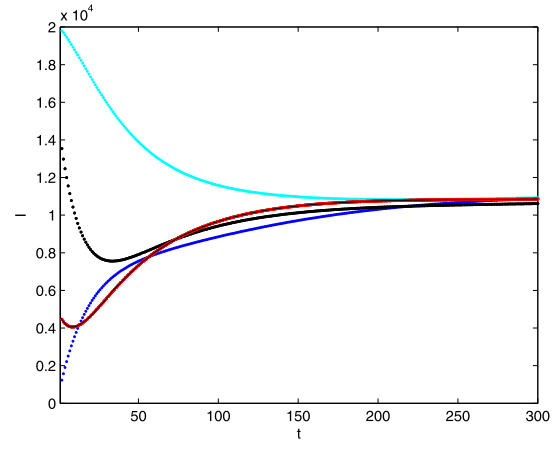

(b) $R_{0}=1.1103, \alpha=0.6$

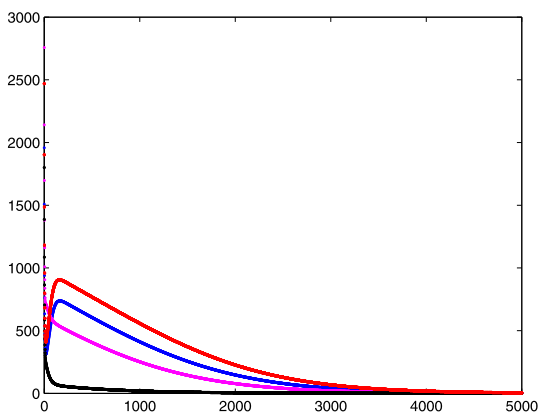

(d) $R_{0}=0.9797, \alpha=0.85$

Figure 7 Time series plots with different initial values.

Time series of $I$ are plotted in Figure 7(a)-(d) showing the disease-free equilibrium and two endemic equilibria. Fix $\alpha=0.5$, then $R_{0}=1.1886>1$; and fix $\alpha=0.6$, then $R_{0}=1.1103>1$. By Theorem 4.1, we have that the disease will be endemic (see Figure 7(a)(b)). Taking $\alpha=0.85$, by (3.1) and (3.11), we can calculate $R_{0}=0.9797, R_{0}^{c}=0.9708$, then $R_{0}^{c}<R_{0}<1$. If initial values close to $E_{0}=(84,1,790,0,0,1,050,367,0)$ are chosen, then the solution of system (2.1) converges to the stable disease-free equilibrium $E_{0}$ (see Figure 7 


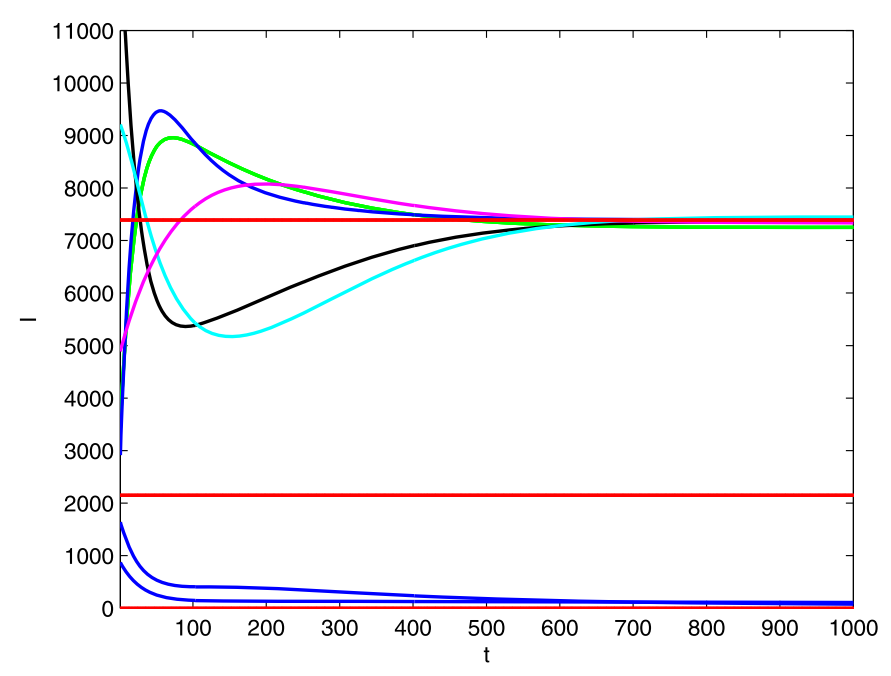

Figure 8 The trajectories of infected psyllid in (2.1) with $\alpha=0.85$ for different selection of initial values.

(d)). Further, if initial values close to endemic equilibria $E_{2}=(99,1,253,172,32,1,042,977$, $7,390)$ are chosen, then the solution of system (2.1) converges to the endemic equilibria $E_{2}$ (see Figure 7 (c)). So the outbreak of HLB depends on the initial size of every population. The disease can die out if the initial values lie in the basin of attraction of $E_{0}$, or it can persist if the initial values lie in the basin of attraction of $E_{2}$. Figure 8 shows that the bigger endemic equilibrium is stable, the smaller is unstable, and the disease-free equilibrium is stable. This clearly shows the co-existence of two locally-asymptotically stable equilibria when $R_{0}<1$.

\section{Conclusions}

In this paper, a deterministic model with bilinear incidence is formulated to study the impact of cross protection on the spread and control of HLB. When we choose appropriate parameters, there exists a backward bifurcation. If $R_{0}>1$, then there is a unique endemic equilibrium and the disease is uniformly persistent. If $R_{0}<1$, there may be two endemic equilibria, and the endemic equilibrium can coexist with the disease-free equilibrium. This illustrates that $R_{0}<1$ cannot ensure the eradication of the disease, and decreasing $R_{0}$ below the sub-threshold $R_{0}^{c}$ would be a propositional control strategy. If $R_{0}^{c}<R_{0}<1$, only when the numbers of infected cases are small enough, it is a sufficient condition to eliminate HLB. Numerical examples are given to demonstrate the effectiveness of the theoretical results.

Our investigations suggest that cross protection and removing infected trees play an important role in controlling the spread of HLB. Cross protection also dramatically affects the disease transmission dynamics. Moreover, increasing the replanting rate is bad for disease control. The result strongly suggests and supports the previous observations [39, 40]. 


\section{Competing interests}

The authors declare that they have no competing interests.

\section{Authors' contributions}

The main idea and theoretical proof of this paper were proposed by LL and SG. Programming of numerical simulation was completed by $Y L$ and $Y G$. All authors read and approved the final manuscript.

\section{Publisher's Note}

Springer Nature remains neutral with regard to jurisdictional claims in published maps and institutional affiliations.

Received: 10 May 2017 Accepted: 7 October 2017 Published online: 06 November 2017

\section{References}

1. Bové, JM: Huanglongbing: a destructive, newly-emerging, century-old disease of citrus. J. Plant Pathol. 88, 7-37 (2006)

2. Taylor, RA, Mordecai, EA, Gilligan, CA, Rohr, JR, Johnson, LR: Mathematical models are a powerful method to understand and control the spread of Huanglongbing. PeerJ 19, 2642 (2016)

3. Jacobsen, K, Stupiansky, J, Pilyugin, SS: Mathematical modeling of citrus groves infected by Huanglongbing. Math. Biosci. Eng. 10, 705-728 (2013)

4. Zhang, M, Guo, Y, Powell, CA, Doud, MS, Yang, C, Duan, Y: Effective antibiotics against "Candidatus Liberibacter asiaticus" in HLB-affected citrus plants identified via the graft-based evaluation. PLoS ONE 9(11), 1-11 (2014)

5. Hoffman, MT, Doud, MS, Williams, L, Zhang, M, Ding, F, Stover, E, Hall, D, Zhang, S, Jones, L, Gooch, M, Fleites, L, Dixon, W, Gabriel, D, Duan, Y: Heat treatment eliminates 'Candidatus Liberibacter asiaticus' from infected citrus trees under controlled conditions. Phytopathology 103, 15-22 (2013)

6. Dutt, M, Barthe, G, Irey, M, Grosser, J: Correction: Transgenic citrus expressing an Arabidopsis NPR1 gene exhibit enhanced resistance against Huanglongbing (HLB; Citrus Greening). PLoS ONE 11(1), 1-17 (2016)

7. Gottwald, TR, Graham, JH, Irey, MS, McCollum, TG, Wood, BW: Inconsequential effect of nutritional treatments on huanglongbing control, fruit quality, bacterial titer and disease progress. Crop Prot. 36, 73-82 (2012)

8. Gottwald, TR, Hall, DG, Kriss, AB, Salinas, EJ, Parker, PE, Beattie, GAC, Nguyen, MC: Orchard and nursery dynamics of the effect of interplanting citrus with guava for huanglongbing, vector, and disease management. Crop Prot. 64, 93-103 (2014)

9. McKinney, HH: Mosaic diseases in the Canary Islands, West Africa and Gibraltar. J. Agric. Res. 39, 557-578 (1929)

10. Zhang, XS, Holt, J: Mathematical models of cross protection in the epidemiology of plant-virus diseases. Phytopathology 91, 924-934 (2001)

11. Rochow, WF, Muller, I, Gildow, FE: Interference between two luteoviruses in an aphid: lack of reciprocal competition. Phytopathology 73, 919-922 (1983)

12. Cui, B, Cui, S, Wang, H, Weng, F, Gong, J: Study of cross protection of citrus tristeza viruse disease on Bendizao mandarin (Citrus succosa). Agric. Life Sci. 31, 433-438 (2005)

13. van Vuuren, SP, van der Vyver, JB, Lutting, M, da Graca, JV: Low incidence of Huanglong-bing fruit symptoms in Valencia sweet orange trees in the presence of a population of Citrus tristeza virus. In: Proc. 4th Conf. IOCV, pp. 373-377. IOCV, Valencia (2000)

14. Hartung, JS, Folimonov, AS, Folimonov, SY, Dawson, WO: H-16 screening of antibacterial peptides in citrus trees for activity against Candidatus Liberibacter asiaticus. In: Second International Citrus Canker and Huanglongbing Research Workshop 64

15. Meng, X, Li, Z: The dynamics of plant disease models with continuous and impulsive cultural control strategies J. Theor. Biol. 266, 29-40 (2010)

16. Meng, X, Song, Z, Chen, L: A new mathematical model for optimal control strategies of integrated pest management. J. Biol. Syst. 15, 219-234 (2007)

17. Zhang, T, Meng, X, Song, Y, Li, Z: Dynamical analysis of delayed plant disease models with continuous or impulsive cultural control strategies. Abstr. Appl. Anal. 2012, 1-25 (2012)

18. Zhao, W, Li, J, Zhang, T, Meng, X, Zhang, T: Persistence and ergodicity of plant disease model with Markov conversion and impulsive toxicant input. Commun. Nonlinear Sci. Numer. Simul. 48, 70-84 (2017)

19. Xia, L, Gao, S, Zou, Q, Wang, J: Analysis of a nonautonomous plant disease model with latent period. Appl. Math. Comput. 223, 147-159 (2013)

20. Gao, S, Xia, L, Wang, J, Zhang, Z: Modelling the effects of cross protection control in plant disease with seasonality. Int. J. Biomath. 10, 1-24 (2017)

21. Zhang, XS, Holt, J, Colvin, J: Mathematical models of host plant infection by helper-dependent virus complexes: why are helper viruses always avirulent. Phytopathology 90, 85-93 (2000)

22. Garba, SM, Gumel, AB, Abu Bakar, NR: Backward bifurcations in dengue transmission dynamics. Math. Biosci. 215 , 11-25 (2008)

23. Abdelrazec, A, Bélair, J, Shan, C, Zhu, H: Modelling the spread and control of dengue with limited public health resources. Math. Biosci. 271, 136-145 (2016)

24. Li, X, Li, W, Ghosh, M: Stability and bifurcation of an SIR epidemic model with nonlinear incidence and treatment. Appl. Math. Comput. 210, 141-150 (2009)

25. Abdelrazec, A, Lenhart, S, Zhu, H: Transmission dynamics of West Nile virus in mosquitoes and corvids and non-corvids. J. Math. Biol. 68, 1553-1582 (2014)

26. Hu, Z, Chang, L, Teng, Z, Chen, X: Bifurcation analysis of a discrete SIRS epidemic model with standard incidence rate. Adv. Differ. Equ. 2016, 155 (2016)

27. Zhang, T, Kang, R, Wang, K, Liu, J: Global dynamics of an SEIR epidemic model with discontinuous treatment. Adv. Differ. Equ. 2015, 361 (2015). doi:10.1186/s13662-015-0695-0

28. Diekmann, O, Heesterbeek, JAP: Mathematical Epidemiology of Infectious Diseases: Model Building, Analysis and Interpretation. Math. Comp. Biol. Wiley, New York (2000) 
29. van den Driessche, P, Watmough, J: Reproduction numbers and sub-threshold endemic equilibria for compartmental models of disease transmission. Math. Biosci. 180, 29-48 (2002)

30. Blayneh, KW, Gumel, AB, Lenhart, S, Clayton, T: Backward bifurcation and optimal control in transmission dynamics of West Nile virus. Bull. Math. Biol. 72, 1006-1028 (2010)

31. Wan, H, Zhu, H: The backward bifurcation in compartmental models for West Nile virus. Math. Biosci. 227, 20-28 (2010)

32. Castillo-Chavez, C, Song, B: Dynamical models of tuberculosis and their applications. Math. Biosci. Eng. 1, 361-404 (2004)

33. Zhao, XQ: Dynamical System in Population Biology. Springer, New York (2003)

34. Zhang, F, Zhao, XQ: A periodic epidemic model in a patchy environment. J. Math. Anal. Appl. 325, 496-516 (2007)

35. Deng, XM: Forming process and basis and technological points of the theory emphasis on control citrus psylla for integrated control Huanglongbing. Chin. Agric. Sci. Bull. 25, 358-363 (2009) (in Chinese)

36. Taylor, RA, Mordecai, E, Gilligan, CA, Rohr, JR, Johnson, LR: Mathematical models are a powerful method to understand and control the spread of Huanglongbing. PeerJ 4, e2642 (2016). doi:10.7717/peerj.2642

37. Liu, YH, Tsai, JH: Effects of temperature on biology and life table parameters of the Asian citrus psyllid, Diaphorina citri kuwayama (Homoptera: Psyllidae). Ann. Appl. Biol. 137, 201-206 (2000)

38. Hall, DG, Wenninger, EJ, Hentz, MG: Temperature studies with the Asian citrus psyllid, Diaphorina citri: cold hardiness and temperature thresholds for oviposition. J. Insect Sci. 11, 83 (2011)

39. Xia, L, Gao, S, Liu, Y, Xie, D: A plant virus disease model with periodic environment and pulse roguing. Stud. Appl. Math. 136, 357-381 (2016). doi:10.1111/sapm.12109

40. Chan, MS, Jeger, MJ: An analytical model of plant virus disease dynamics with roguing and replanting. J. Appl. Ecol. 31, 413-427 (1994)

\section{Submit your manuscript to a SpringerOpen ${ }^{\circ}$ journal and benefit from:}

- Convenient online submission

- Rigorous peer review

- Open access: articles freely available online

- High visibility within the field

- Retaining the copyright to your article

Submit your next manuscript at $\boldsymbol{~ s p r i n g e r o p e n . c o m ~}$ 